\title{
Quantitative Nanoscale Absorption Mapping: A Novel Technique To Probe Optical Absorption of Two-Dimensional Materials
}

\author{
Marco Negri, ${ }^{\dagger}$ Luca Francaviglia, ${ }^{\dagger}$ Dumitru Dumcenco, ${ }^{\dagger, \dagger, \nabla}$ Matteo Bosi, ${ }^{\S}$ Daniel Kaplan, \\ Venkataraman Swaminathan, ${ }^{\|, \bigcirc}$ Giancarlo Salviati, ${ }^{\S}$ Andras Kis, ${ }^{\ddagger}$ Filippo Fabbri, ${ }^{*}, \perp$ \\ and Anna Fontcuberta i Morral ${ }^{*},, \#$ (C)
}

${ }^{\dagger}$ Institute of Materials, Faculty of Engineering, École Polytechnique Fédérale de Lausanne, 1015 Lausanne, Switzerland

${ }^{\ddagger}$ Electrical Engineering Institute, École Polytechnique Fédérale de Lausanne (EPFL), CH-1015 Lausanne, Switzerland

${ }^{\S}$ Institute for Materials for Electronics and Magnetism (IMEM-CNR), Parco Area delle Scienze 37/A, 43124 Parma, Italy

${ }$ Fuze Precision Armaments and Technology Directorate, U.S. Army RDECOM-ARDEC, Picatinny Arsenal, New Jersey 07806, United States

${ }^{\perp}$ NEST, Istituto Nanoscienze-CNR, Scuola Normale Superiore, Piazza San Silvestro 12, 56127 Pisa, Italy

\#Institute of Physics, Faculty of Basic Sciences, École Polytechnique Fédérale de Lausanne, 1015 Lausanne, Switzerland

Supporting Information

\begin{abstract}
Two-dimensional semiconductors, in particular transition metal dichalcogenides and related heterostructures, have gained increasing interest as they constitute potential new building blocks for the next generation of electronic and optoelectronic applications. In this work, we develop a novel nondestructive and noncontact technique for mapping the absorption properties of $2 \mathrm{D}$ materials, by taking advantage of the underlying substrate cathodoluminescence emission. We map the quantitative absorption of $\mathrm{MoS}_{2}$ and $\mathrm{MoSe}_{2}$ monolayers, obtained on sapphire and oxidized silicon, with nanoscale resolution. We extend our technique to the characterization of the absorption properties of $\mathrm{MoS}_{2} / \mathrm{MoSe}_{2}$ van der Waals

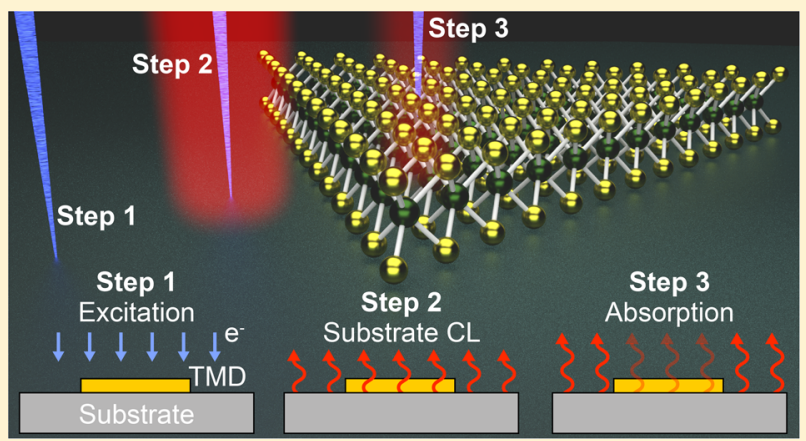
heterostructures. We demonstrate that interlayer excitonic phenomena enhance the absorption in the UV range. Our technique also highlights the presence of defects such as grain boundaries and ad-layers. We provide measurements on the absorption of grain boundaries in monolayer $\mathrm{MoS}_{2}$ at different merging angles. We observe a higher absorption yield of randomly oriented monolayers with respect to $60^{\circ}$ rotated monolayers. This work opens up a new possibility for characterizing the functional properties two-dimensional semiconductors at the nanoscale.
\end{abstract}

KEYWORDS: Cathodoluminescence, absorption mapping, 2D materials, transition metal dichalcogenides, grain boundaries, van der Waals heterostructure

\begin{abstract}
A tomically thin semiconductors hold the promise of complementing graphene in many different applications, where the lack of a band gap hampers its use. Single-layer transition metal dichalcogenides (TMDs) are the most studied class of $2 \mathrm{D}$ semiconductors to date. ${ }^{1-4}$ They have been successfully applied in nanoelectronic devices like field-effect transistors, ${ }^{5}$ nonvolatile memories, ${ }^{6}$ or logic circuits. ${ }^{7,8}$ The direct gap of single-layer TMDs, in the visible and nearinfrared ranges of the electromagnetic spectrum, ${ }^{9,10}$ has also triggered the interest in its optical properties with the perspective of a new generation of optoelectronic devices. ${ }^{11}$

Looking beyond the study of a single material, isolated atomic planes can also be assembled into heterostructures, often referred to as "van der Waals" (vdW) heterostructures made layer by layer in a precisely chosen sequence without the constraints imposed by epitaxy such as crystal-lattice match or
\end{abstract}

chemistry compatibility. ${ }^{12}$ The combination of $2 \mathrm{D}$ materials offers a truly unprecedented potential to discover new physical phenomena or to engineer novel electronic or optical functionalities. ${ }^{12}$ For instance, it has been shown that the encapsulation of $\mathrm{MoS}_{2}$ and $\mathrm{MoSe}_{2}$ monolayers by h-BN results in a significant improvement of the optical yield and in the narrowing of the excitonic emission line width. ${ }^{13,14}$

The combination of single-layer TMDs provides new electronic and optoelectronic functionalities, such as the formation of atomically thin $\mathrm{p}-\mathrm{n}$ junctions ${ }^{15}$ and ultrafast charge separation. ${ }^{16}$ The interlayer interaction in TMD-based $\mathrm{vdW}$ heterostructures has resulted in a manifold of novel

Received: October 18, 2019

Revised: December 24, 2019

Published: December 24, 2019 


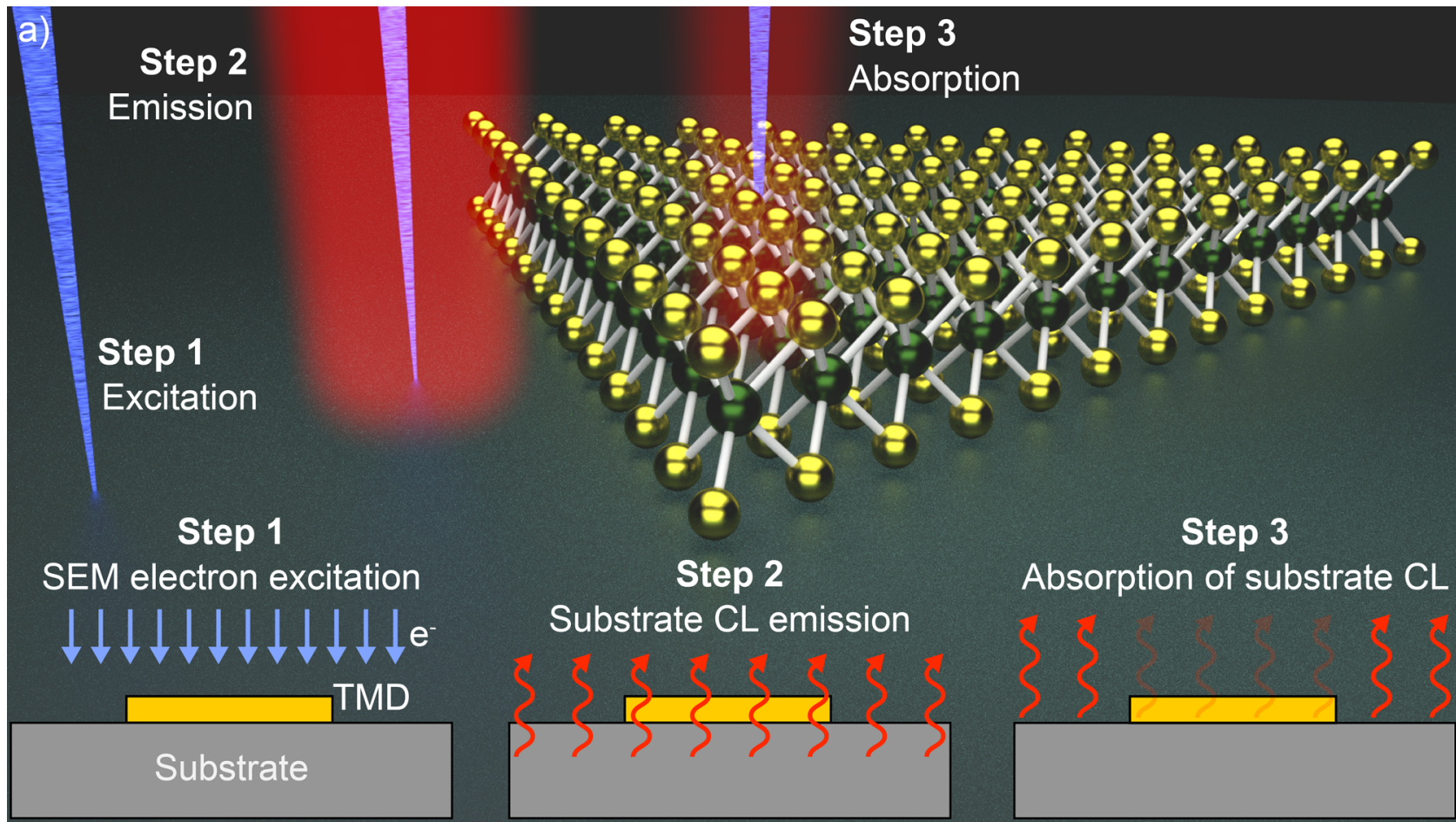

b)

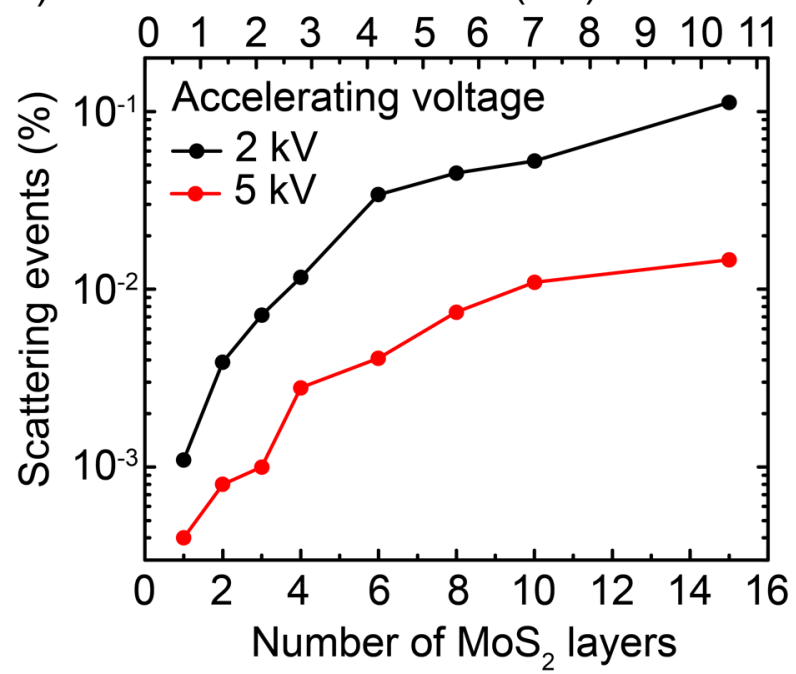

c)

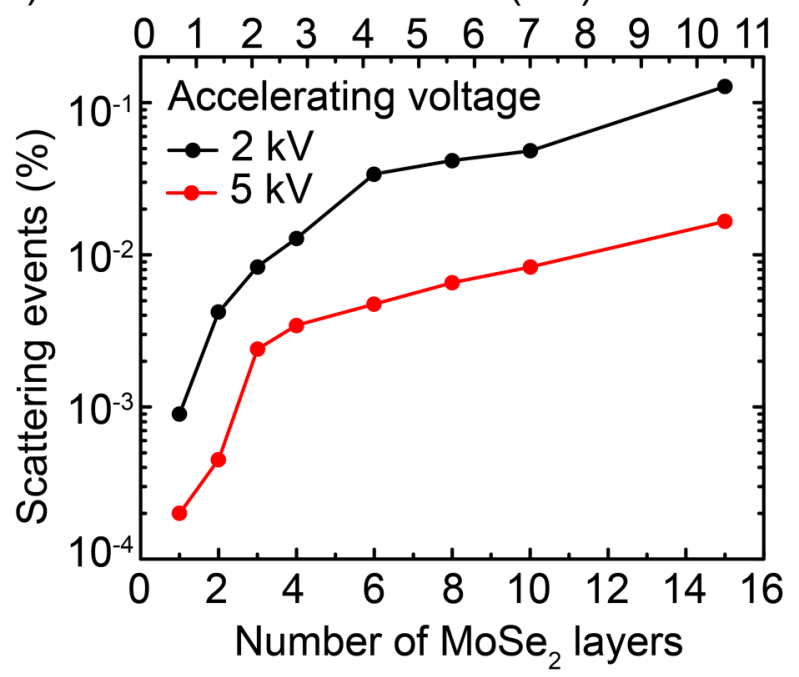

Figure 1. (a) Sketch of the working principle of the technique. Step 1, a focused electron beam rasters the sample surface; step 2, the electron beam excites cathodoluminescence emission of both the substrate and the flake; due to the limited interaction volume, the emission from the flake is too weak, and it is not detected; step 3, the flake absorbs part of the substrate CL emission, which results in the QNAM contrast between bare and ML covered regions of the substrate. (b, c) Scattering events (as percentage on the total) in $\mathrm{MoS}_{2}$ and $\mathrm{MoSe}_{2}$, respectively, as a function of the layer thickness and for the accelerating voltage of 2 and $5 \mathrm{kV}$.

optical phenomena. For instance, a new type of exciton where the electron and the hole are spatially located in different layers, named interlayer exciton, has been demonstrated. ${ }^{17-20}$ The possible formation of the interlayer exciton is promoted by the staggered band alignment of the TMD semiconducting single layer. ${ }^{19,21}$ These properties have already been successfully applied to optoelectronic devices for light emission or absorption. $^{22}$

Recently, the effect of the twist angle between the different single layers on the interlayer exciton energy and yield has been reported. ${ }^{23}$ As an example, the interlayer coupling of excitons in $\mathrm{MoS}_{2}$ bilayers is strongly dependent on the twist angles between the two monolayers (MLs). ${ }^{24}$ In $\mathrm{MoS}_{2} / \mathrm{MoSe}_{2}$ heterostructures, the characteristics of the intralayer excitons strongly depend on the details of the Moire pattern. The potential fluctuations resulting from the locally different atomic registries split the trion and exciton transitions of $\mathrm{MoSe}_{2}$ and $\mathrm{MoS}_{2}$ into two peaks related to optically active local minima of the Moiré potential. ${ }^{25,26}$

Scanning confocal spectroscopic techniques, namely, photoluminescence and absorption spectroscopies, have provided important insights on the optical properties of TMD single layers and related vdW heterostructures at the micrometric scale. In particular, scanning PL spectroscopy has unveiled 
quantum confinement induced direct-to-indirect band gap transition, 9,10 photoluminescence emission from charged excitons, ${ }^{27,28}$ and valley-polarized photoluminescence yield. $^{29-31}$ In addition, scanning confocal photoluminescence spectroscopy has elucidated the presence of grain boundaries. $^{32}$ However, photoluminescence spectroscopy is an inefficient technique to study the optical properties of indirect band gap few-layer TMDs. Finally, spatially resolved absorption mapping has mainly demonstrated the evolution of the absorption properties of TMDs increasing the number of layers in exfoliated flakes. ${ }^{33,34}$

These characterization techniques are limited to a spatial resolution of few hundred nanometers at least. In order to overcome the diffraction limit, the near-field regime is required. Near-field photoluminescence imaging of transition metal dichalcogenides has been mainly employed to study the optical properties of nanometric features of monolayers, as extended defects. Near-field photoluminescence imaging is, for instance, employed in the analysis of pyramidal ad-layer defects and disordered edges in $\mathrm{ML} \mathrm{MoS}$, showing a similar behavior as an intensity decrease and concurrent red-shift of the PL emission. $^{35,36}$

A particular class of near-field optical techniques is the tipenhanced PL spectroscopy (TEPS), which merges a scanning probe system with an optical spectrometer. TEPS allows mapping of photoluminescence with spatial resolution in the order of tens of nanometers. ${ }^{37}$ This high spatial resolution is possible due to the amplification of the optical signal by means of a plasmonic metal tip of the scanning probe system. The main inconvenience of this technique is the long acquisition times. TEPS experiments are mainly employed to study the optical properties at the nanoscale, such as the PL yield in $\mathrm{MoS}_{2}$ bilayer terraces or strain in $\mathrm{WSe}_{2} .{ }^{38,39}$

Cathodoluminescence $(\mathrm{CL})$ is the light emission of a material excited by an electron beam. Although CL spectroscopy and mapping have been employed to explore the light emission properties of ML TMDs, this technique suffers from some limitations in the study of two-dimensional materials due to the limited interaction volume of the primary electron beam with an atomically thin sample. In fact, the direct CL emission of TMDs has been only reported in the case of multilayer $\mathrm{MoS}_{2}$ flakes $^{40}$ and TMD single layers encapsulated in h-BN flakes that act as a reservoir of charges injected in the TMD single layer. $^{41}$

In this work, we propose a novel noncontact and nondestructive optical technique for the study of $2 \mathrm{D}$ materials at the nanoscale, named quantitative nanoscale absorption mapping (QNAM). The particular approach of QNAM is to probe the absorption properties of a $2 \mathrm{D}$ material using the $\mathrm{CL}$ emission of the underlying substrate as the light source. Figure la shows the functioning principle of QNAM. A bulk substrate topped with a two-dimensional layer is excited by an electron beam. The substrate emits light via a cathodoluminescence process. The emitted light is partially absorbed by the $2 \mathrm{D}$ material, and it is collected by a state-of-the-art CL system. A direct mapping of the substrate CL provides information on light absorption by the $2 \mathrm{D}$ crystal with nanoscale resolution. A video summarizing the physical process is shown in the Supporting Information. The result is a two-dimensional map of the absorption that can reveal features difficult to detect using other electron microscopy or scanning confocal optical techniques. The main limitation to the spatial resolution is the interaction volume of the electron beam with the substrate.
In addition to the straightforward generation of absorption maps of TMDs with nanoscale spatial resolution, QNAM experiments can be carried out on the growth substrate, without any transfer procedure. Transferring may not only introduce strain or even damage the TMD flake but also alter the surrounding dielectric environment to which $2 \mathrm{D}$ materials are highly sensitive. ${ }^{42}$ In order to develop this novel technique, we carried out preliminary experiments on $\mathrm{MoS}_{2}$ and $\mathrm{MoSe}_{2}$ single layers grown by chemical vapor deposition on different substrates, namely, $\mathrm{SiO}_{2} / \mathrm{Si}$ and sapphire. Besides a qualitative mapping, QNAM also allows the extraction of quantitative information. We therefore studied in quantitative terms the absorption properties of the grain boundaries of $\mathrm{MoS}_{2}$ single layers with different misorientation angles. In particular, QNAM reveals that the absorption contrast of the $\mathrm{MoS}_{2}$ / $\mathrm{MoSe}_{2}$ vdW heterostructure is 4 times higher with respect to the single layers, demonstrating a strong interlayer interaction.

Results and Discussion. The two main requisites for QNAM are a relatively weak interaction of the twodimensional material with the primary electron beam and a significant CL emission of the substrate which should overlap with the absorption of the $2 \mathrm{D}$ material. In addition, pointdefects related CL emission are preferable in order to avoid possible interactions between the substrate and the monolayer. We carried out Monte Carlo simulations in order to evaluate the interaction of the primary electron beam with monolayers $\mathrm{MoS}_{2}$ and $\mathrm{MoSe}_{2}$. Monte Carlo simulations provide samplespecific electron penetration depth and interaction volume by modeling the probability of each scattering event of the primary electrons in the sample. For instance, in the case of a $\mathrm{MoS}_{2}$ or $\mathrm{MoSe}_{2}$ monolayer grown on sapphire, it is possible to evaluate the ratio of electron scattering events in the twodimensional crystal and in the substrate, respectively. Figure $1 b, c$, respectively, shows the probability of scattering events in $\mathrm{MoS}_{2}$ and $\mathrm{MoSe}_{2}$ as a function of the number of the layers and for accelerating voltages of 2 and $5 \mathrm{kV}$. The probability of scattering events in the TMD is expressed as a percentage of the total, that is, as a fraction of the sum of scattering events in both the $2 \mathrm{D}$-crystal and the substrate The accelerating voltage is an important parameter for CL because it drastically changes the electron penetration depth. However, at both 2 and $5 \mathrm{kV}$ the simulations demonstrate that the interaction of the primary electron beam with the two-dimensional crystal is negligible compared to the interaction with the substrate. As a consequence, also the CL intensity from the TMDs is negligibly weak. For instance, at $2 \mathrm{kV}$ the percentage of scattering events in both $\mathrm{MoS}_{2}$ and $\mathrm{MoSe}_{2}$ is still less than $0.01 \%$ for a relatively thick TMD trilayer. Further details on the electron beam penetration are reported for 1 and $5 \mathrm{MoS}_{2}$ layers in Figures S1 and S2 of the Supporting Information.

In this section, we will provide the QNAM measurements and analysis on $\mathrm{MoS}_{2}$ and $\mathrm{MoSe}_{2}$ MLs on sapphire substrates carried out with an accelerating voltage of $5 \mathrm{kV}$. Sapphire is chosen as the TMD growth substrate because it provides a route to control the orientation of TMD domains, with the potential to achieve high-quality TMD MLs. ${ }^{43}$ Moreover, it is well-established that single crystalline sapphire has an intense CL peak in the UV range $(332 \mathrm{~nm}, 3.84 \mathrm{eV})$, ascribed to the emission of the $\mathrm{F}^{+}$center, an oxygen vacancy with a single trapped electron. ${ }^{44,45}$ The UV emission allows the study of the optical absorption of TMDs in the UV range, which would be a rather challenging analysis to perform with traditional spectroscopy approaches due to technical limitations, like the 

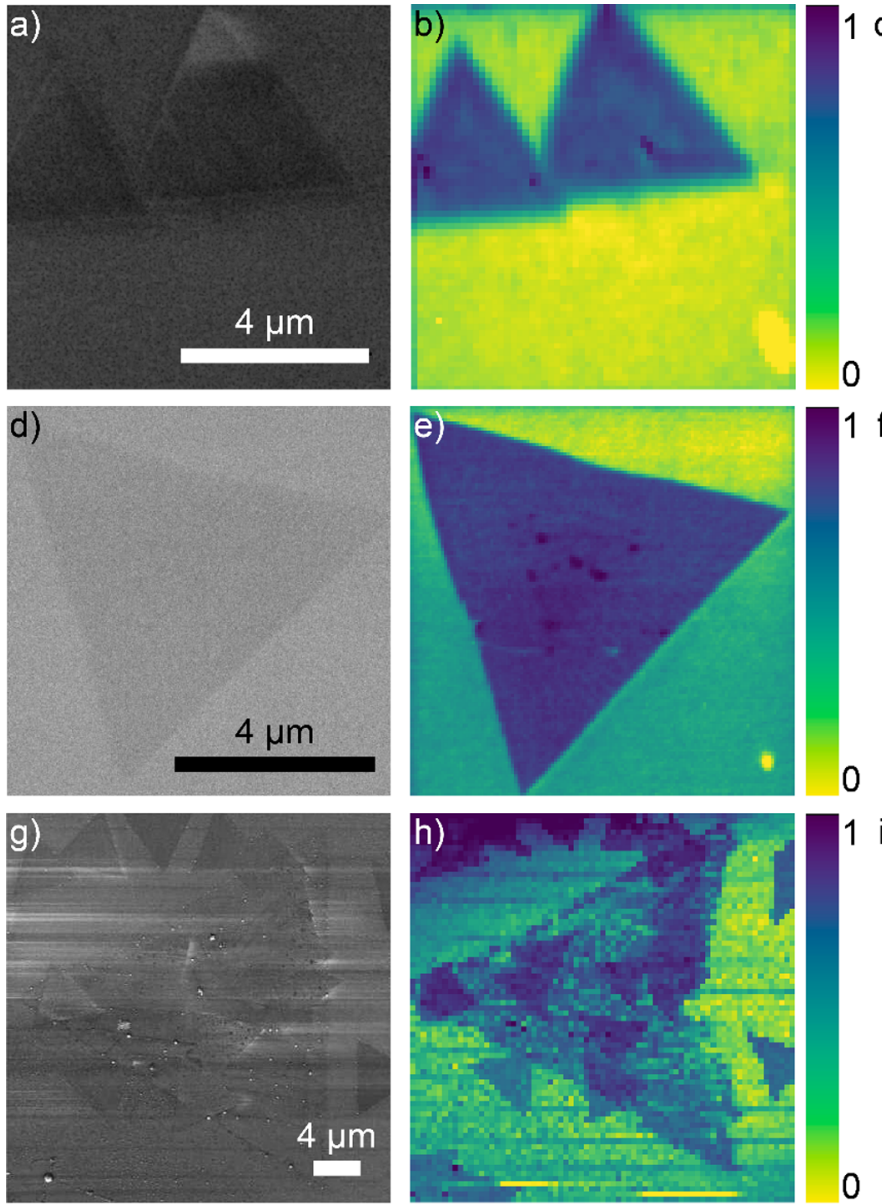

$1 \mathrm{c})$

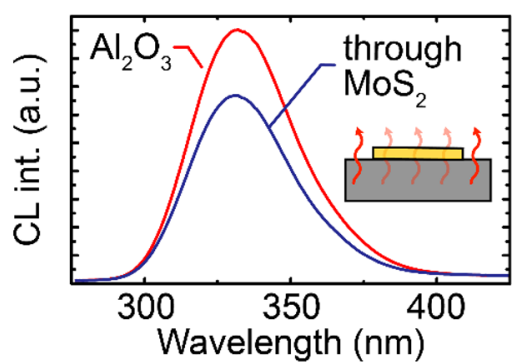

f)

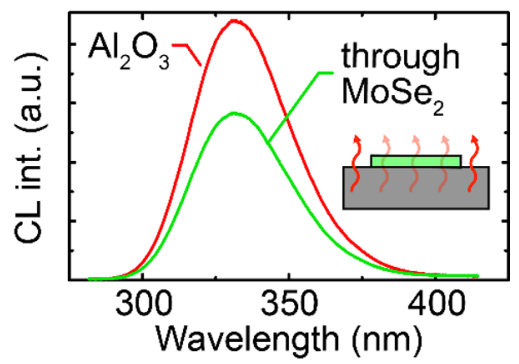

i)

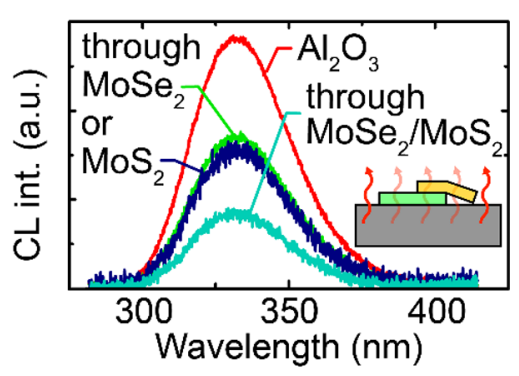

Figure 2. (a) Secondary electron image at $5 \mathrm{kV}$ of $\mathrm{MoS}_{2} \mathrm{ML}$ on sapphire substrate. (b) QNAM map of MoS $\mathrm{M}_{2} \mathrm{ML}$. (c) CL spectra from the bare substrate and from the $\mathrm{MoS}_{2} \mathrm{ML}$ area. (d) Secondary electron image at $5 \mathrm{kV}$ of $\mathrm{MoSe}_{2}$ flake on $\mathrm{Al}_{2} \mathrm{O}_{3}$. (e) QNAM map of the MoSe $e_{2}$. (f) CL spectra from the bare substrate and from the $\mathrm{MoSe}_{2} \mathrm{ML}$ area ML. (g) Secondary electron image at $5 \mathrm{kV}$ of a van der Waals heterostructure composed of $\mathrm{MoSe}_{2} \mathrm{ML}$ (larger) and several $\mathrm{MoS}_{2}$ MLs (smaller) on sapphire. (h) QNAM map of the vdW heterostructures. (i) CL spectra of bare substrate, $\mathrm{MoS}_{2} \mathrm{ML}, \mathrm{MoSe}_{2} \mathrm{ML}$, and $\mathrm{MoS}_{2}-\mathrm{MoSe}_{2}$ vdW heterostructures.

light absorption from the substrate or from the cuvette in the case of single flakes ${ }^{34}$ or exfoliated TMDs solutions, ${ }^{46}$ respectively. Normally the high energy absorption of TMDs has been analyzed by means of spectroscopic ellipsometry in order to study the real and imaginary parts of the dielectric constant. ${ }^{47}$ Figure 2a shows the SEM image of $\mathrm{MoS}_{2}$ flakes synthesized on sapphire. The SE image does not show particular features in the flake area. The QNAM contrast map of the $\mathrm{MoS}_{2}$ monolayer is homogeneous throughout the flake except for the presence of nanometric features, not detected in the SE image, with a higher local absorption. These features can be related to nanoscale terraces of additional layers or to pyramidal ad-layer defects. Figure $2 \mathrm{c}$ reports the CL spectra of the sapphire emission acquired over the area topped with $\mathrm{MoS}_{2}$ MLs (blue line) and over the bare regions (red curve). The comparison of the integrated intensities allows the evaluation of the QNAM contrast. The method for the evaluation of the QNAM contrast is reported in the Methods section. The QNAM contrast of a $\mathrm{MoS}_{2}$ single layer in the UV range is $7.2 \%$. In order to compare the absorption and emission properties, the confocal PL intensity map of a $\mathrm{MoS}_{2}$ single-layer is reported in the Supporting Information, Figure S3. In order to prove the versatility of QNAM we also analyze $\mathrm{MoSe}_{2}$ monolayers, a two-dimensional semiconductor with narrower band gap $(1.58 \mathrm{eV}, 785 \mathrm{~nm})^{48}$ compared with
$\mathrm{MoS}_{2}$. Figure $2 \mathrm{~d}$ shows the SEM image of a $\mathrm{MoSe}_{2}$ flake synthesized on a sapphire substrate. Similarly to the case of $\mathrm{MoS}_{2}$, the QNAM map of $\mathrm{MoSe}_{2}$, reported in Figure 2e, shows a homogeneous contrast in the monolayer area. Nevertheless, it highlights a micrometric bilayer terrace at the center of the flake and additional pyramidal ad-layer defects with higher absorption contrast. Figure $2 \mathrm{f}$ shows the CL spectra of the area topped with the flake (green line) and of the bare substrate (red line). We detect a similar QNAM contrast of $\mathrm{MoSe}_{2}$ monolayer, $7.4 \%$ in comparison with $\mathrm{MoS}_{2}$, indicating comparable absorption efficiency of $\mathrm{MoSe}_{2}$ monolayers.

The optical properties of TMD-based vdW heterostructures $^{17,18}$ have demonstrated the peculiarity of interlayer phenomena. Here, we show the potential of QNAM in the analysis of $\mathrm{vdW}$ heterostructures and related interlayer phenomena by mapping the stacking of $\mathrm{MoS}_{2}$ MLs on top of $\mathrm{MoSe}_{2}$ monolayers. Figure $2 \mathrm{~g}$ shows the SEM images of a $\mathrm{MoS}_{2} / \mathrm{MoSe}_{2}$ heterostructure on sapphire substrate. The MLs with an average lateral size of $5 \mu \mathrm{m}$ are the $\mathrm{MoS}_{2}$ flakes, while the larger flakes are $\mathrm{MoSe}_{2} \mathrm{MLs}$ (average lateral size $40 \mu \mathrm{m}$ ). The $\mathrm{MoS}_{2}$ MLs are transferred with a standard polymerassisted process, as described in the Methods section. The corresponding QNAM map is reported in Figure $2 \mathrm{~h}$ and shows higher absorbance in the heterostructure areas with respect to the areas covered by only one kind of TMD. QNAM allows a 
clear imaging of the flakes without any possible artifact due to charging effect of the substrate, as made evident in the SE image of the vdW heterostructure reported in Figure $2 \mathrm{~g}$. Figure $2 \mathrm{i}$ reports the CL spectra from different areas of the map. The comparison of the CL spectra further corroborates that the absorption is higher through the vdW heterostructure than through the single $\mathrm{MoS}_{2}$ and $\mathrm{MoSe}_{2}$ monolayers. The QNAM contrast in the case of the $\mathrm{MoS}_{2} / \mathrm{MoSe}_{2}$ heterostructure is $19.2 \%$, while the contrasts of the single MLs are $7.4 \%$ and $7.3 \%$ for $\mathrm{MoS}_{2}$ and $\mathrm{MoSe}_{2}$, respectively. This demonstrates that the absorption in the UV range is deeply affected by interlayer phenomena causing the unexpected increase of the absorption yield of more than 2 times. This increase of the absorption yield could be related with the interlayer effect of high-energy excitonic states (i.e., the $\mathrm{C}$ exciton or $\mathrm{E}$ states in $\mathrm{MoS}_{2}$ and $\left.\mathrm{MoSe}_{2}\right) .{ }^{47,49}$ The $\mathrm{C}$ exciton absorption occurs at $428-442 \mathrm{~nm}$ $(2.9-2.8 \mathrm{eV})$ and at $478-496 \mathrm{~nm}(2.5-2.6 \mathrm{eV})$ in the case of $\mathrm{MoS}_{2}$ and $\mathrm{MoSe}_{2}$ MLs, respectively. ${ }^{50,51}$ The comparison with the sapphire $\mathrm{F}+$ emission, centered at $323 \mathrm{~nm}(3.8 \mathrm{eV})$, reveals that the enhanced absorption can be due to high energy states, as the $\mathrm{E}_{2}$ reported in the case of $\mathrm{MoS}_{2}$ MLs by spectral ellipsometry. ${ }^{47}$

Grain boundaries in monolayer TMDs have unique atomic defect structures and band dispersion relations that depend on the interdomain misorientation angle. Different works have demonstrated the importance of the angle dependency on the optical and electrical transport properties at grain boundaries in monolayer $\mathrm{MoS}_{2}{ }^{32,52,53}$ In order to evaluate the UV absorption properties of grain boundaries in monolayer $\mathrm{MoS}_{2}$ as a function of different orientation angles, we perform the QNAM measurements directly on a sapphire substrate. In Figure 3 we show the comparison between QNAM maps of grain boundaries in $\mathrm{MoS}_{2}$ MLs with two misorientation angles, namely, $29^{\circ}$ and $60^{\circ}$. Aberration-corrected high-resolution TEM (HRTEM) studies have previously reported that a misorientation angle of $60^{\circ}$ gives rise to mirror twin boundaries (MTBs); meanwhile, lower misorientation angles
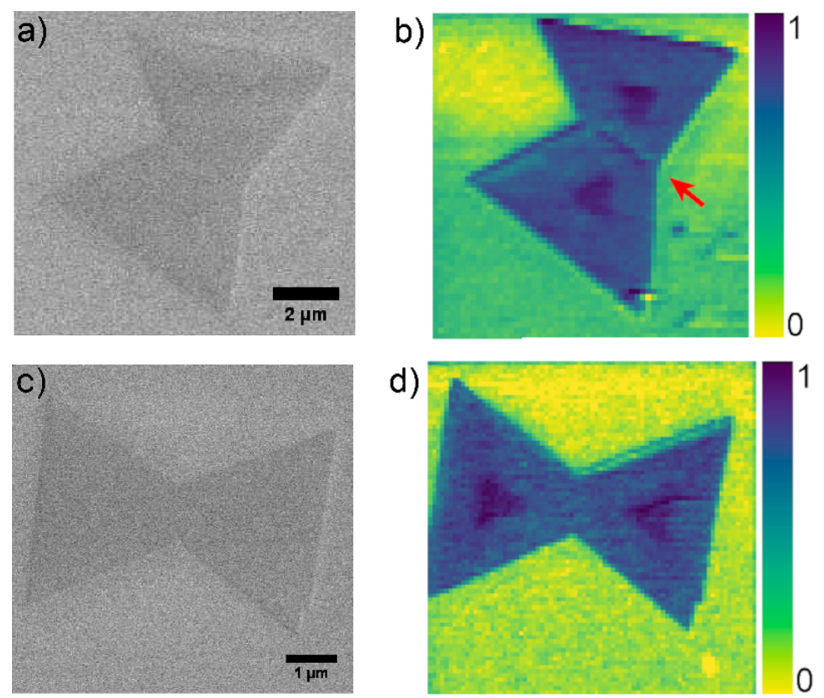

Figure 3. (a) SE image and (b) QNAM map; the red arrow highlights the QNAM contrast of the tilted grain boundaries between MLs with a misorientation angle of $25^{\circ}$. (c) SE image and (d) QNAM map of two $\mathrm{MoS}_{2} \mathrm{MLs}$ with a misorientation angle of $60^{\circ}$ on sapphire substrate. cause tilted boundaries (TBs) between the single crystalline $\mathrm{MoS}_{2}$ domains. The tilted boundaries can be classified in a low (high) angle in the case of a misorientation angle lower (higher) than $13^{\circ}$. The SE image of two $\mathrm{MoS}_{2}$ MLs with a misorientation angle of $25^{\circ}$ is shown in Figure $3 \mathrm{a}$, and the corresponding QNAM map is reported in Figure $3 \mathrm{~b}$. The QNAM map reveals that the grain boundary between the two flakes (highlighted with a red arrow) has a local higher absorption yield with a QNAM contrast of $7.7 \%$. A highresolution line profile analysis of the tilted grain boundaries is reported in Figure S4 of the Supporting Information. The average QNAM contrast of the $\mathrm{MoS}_{2}$ MLs is 7\% in agreement with the results reported in Figure 2. The QNAM analysis reveals the presence of micrometric bilayer terrace with a QNAM contrast of $9 \%$, at the center of each $\mathrm{MoS}_{2}$ monolayers, not detected by the SE imaging. An additional QNAM analysis of $25^{\circ}$ misoriented $\mathrm{MoS}_{2}$ monolayers is presented in Figure S5 of the Supporting Information. Even if the misorientation angle is similar, the coalescence of the MLs occurs at lateral edges instead of the triangle vertex, as reported in Figure 3. We turn now to the direct comparison of the QNAM analysis in the case of two $\mathrm{MoS}_{2}$ single crystalline domains with a misorientation angle of $60^{\circ}$ (Figure 3f). The QNAM map does not show any optical contrast in correspondence of the mirror twin boundaries.

Atomic force microscopy analysis and confocal PL mapping of MLs with similar misorientation angles are reported in Figures S6 and S7. All the CL spectra for the QNAM contrast evaluation are reported in the Supporting Information, Figure S8. The main difference between the mirror twin boundaries and tilted boundaries is the crystalline structure of the different defects. Both classes of grain boundaries are formed by aligned dislocation cores along the $[11-20]$ direction. However, mirror twin boundaries consist of sequences of rings with 8 and 4 alternated Mo and $S$ atoms. ${ }^{32}$ Meanwhile, the structures of tilted boundaries are based on 5-7 rings of Mo and S atoms with the peculiar formation of a $S-S$ bond at the center of the ring structure. ${ }^{53}$ As previously elucidated by density functional theory simulations, ${ }^{54}$ mirror twin and tilted grain boundaries modify the band structure of $\mathrm{ML} \mathrm{MoS}$. Typically, the localized deep-level states related to tilted boundaries act as sinks for carriers, while the mirror twin boundaries have metallic conductivity. Unfortunately, there are currently no calculations on the modification of the band structure at energies in the spectral range analyzed in this work (about $4 \mathrm{eV}$ ).

In order to explore the use of QNAM in different spectral ranges, we carry out QNAM experiments with other substrates. $300 \mathrm{~nm}$ thick $\mathrm{SiO}_{2} /$ silicon is the most commonly used growth substrate for the synthesis of TMD monolayer by different techniques. ${ }^{55}$ In this case, the growth results in randomly oriented $\mathrm{MoS}_{2}$ monolayer domains due to the amorphous nature of thermal $\mathrm{SiO}_{2}$ and its relatively high surface roughness. Nevertheless, $\mathrm{SiO}_{2}$ substrates have an intense CL emission due to several-point-defects-related radiative recombination. The standard CL emission spectrum of amorphous silicon dioxide is well-established. ${ }^{56}$ Three peaks can be identified in the emission spectrum: oxygen-deficiency center (ODC II, $\equiv \mathrm{Si}-\mathrm{Si} \equiv$ ) centered at $460 \mathrm{~nm}(2.7 \mathrm{eV}$ ), selftrapped exciton located at $540 \mathrm{~nm}(2.3 \mathrm{eV})$, and nonbridging oxygen hole center, NBOHC ( $\left.\equiv \mathrm{Si}-\mathrm{O}^{*}\right)$, with the peak positioned at $652 \mathrm{~nm}(1.9 \mathrm{eV})$. We mainly concentrate on the 540 and $652 \mathrm{~nm}$ peaks, because these two emissions are close in energy with the $\mathrm{MoS}_{2} \mathrm{~B}$ and A excitons, respectively. ${ }^{34}$ 
a)

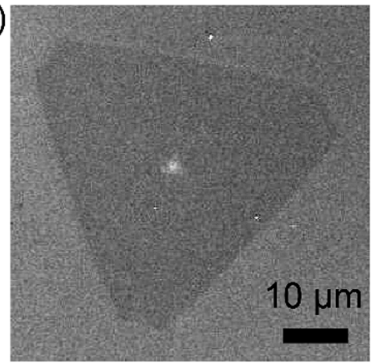

b)

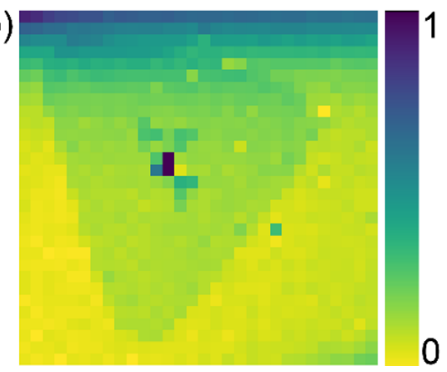

c)

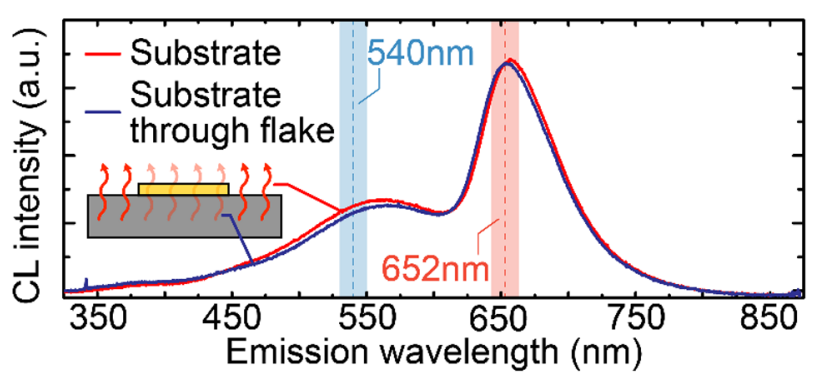

d)
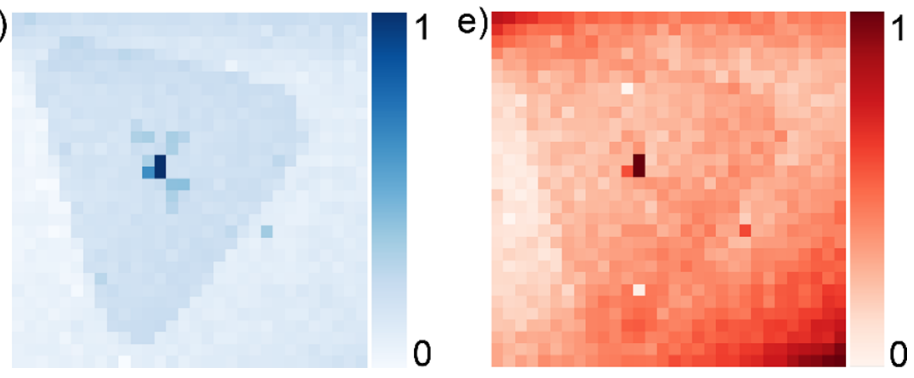

Figure 4. (a) Secondary electron image at $5 \mathrm{kV}$ of a $\mathrm{MoS}_{2}$ flake on $\mathrm{SiO}_{2} / \mathrm{Si}$ substrate. (b) Panchromatic QNAM map. (c) Comparison between the CL emission spectra of the bare substrate (red curve) and the $\mathrm{MoS}_{2} \mathrm{ML}$ (blue curve). (d, e) QNAM monochromatic maps at, respectively, 540 and $652 \mathrm{~nm}$ with a $20 \mathrm{~nm}$ bandwidth; the chosen wavelengths and their bandwidth are indicated in part c.
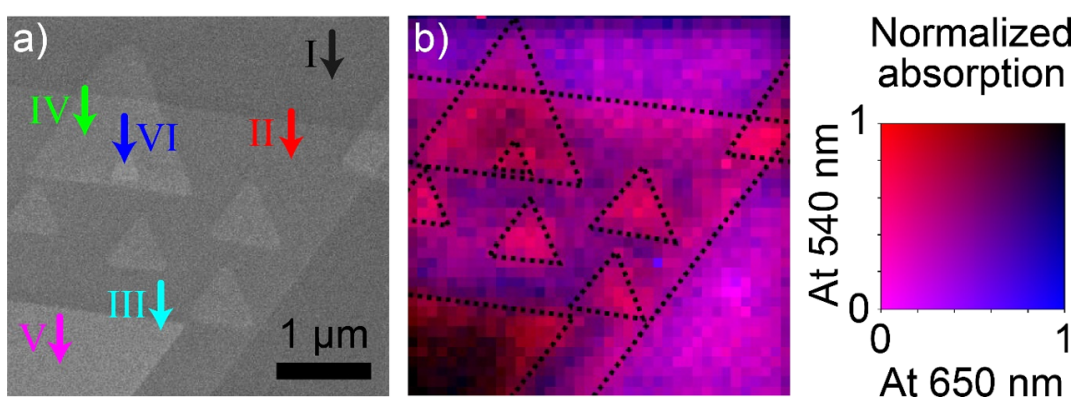

Figure 5. (a) Secondary electron image at $5 \mathrm{kV}$ of superimposed $\mathrm{MoS}_{2}$ flake $\mathrm{SiO}_{2} / \mathrm{Si}$ substrate. (b) Corresponding absorption map at 540 and 650 $\mathrm{nm}(20 \mathrm{~nm}$ bandwidth for each wavelength). The dashed lines are guides to the eye of the superimposed layers; the arrows indicate the areas where the CL spectra (Figure S11) are acquired for the QNAM contrast evaluation. In particular, the bare substrate and the MoS ${ }_{2} \mathrm{ML}_{\text {are indicated by a }}$ black (I) and red (II) arrow, respectively. $0^{\circ}$ and $60^{\circ}$ twisted $\mathrm{MoS}_{2}$ bilayer homostructures are indicated by cyan (III) and green (IV) arrows, respectively. The trilayer terraces, twisted by $0^{\circ}$ and $60^{\circ}$, are highlighted by purple (V) and blue (VI) arrows.

Because of the presence of distinct peaks in the case of the $\mathrm{SiO}_{2} / \mathrm{Si}$ substrate, the QNAM analysis can be carried out in two different modes. In the panchromatic mode the QNAM map is obtained by the CL signal integration over the whole spectral range, i.e., from 350 to $850 \mathrm{~nm}$. In the monochromatic mode, the QNAM map is obtained by integrating the $\mathrm{CL}$ signal of a narrower spectral region, corresponding to the position of a single peak (for further details see the Methods section).

Figure 4a shows the SEM image of an $\mathrm{ML} \mathrm{MoS}$ obtained on a $\mathrm{SiO}_{2} / \mathrm{Si}$ substrate. The bright contrast feature at the center of the flake is due to an ad-layer defect indicating the seeding point of a further monolayer. Figure $4 \mathrm{~b}$ shows the corresponding QNAM panchromatic map of the $\mathrm{MoS}_{2}$ monolayer performed with an accelerating voltage of $5 \mathrm{kV}$. QNAM measurements performed with a $2 \mathrm{kV}$ on $\mathrm{MoS}_{2} \mathrm{ML}$ on $\mathrm{SiO}_{2} / \mathrm{Si}$ is reported in the Supporting Information, Figure S9. The QNAM panchromatic map reports a homogeneous contrast for the $\mathrm{MoS}_{2}$ monolayer except for the center of the map where there is evidence of bilayer terraces with a slightly darker contrast in comparison with the ML, close to the 3dimentional seed of the $\mathrm{MoS}_{2}$ crystal. The comparison between the CL spectra obtained on the bare substrate and on the flake area is reported in Figure 4c, highlighting the two bands of interest. The $\mathrm{MoS}_{2}$ absorption of the substrate CL affects all the analyzed range of wavelength. Moreover, there is a different absorption yield for the emission at $540 \mathrm{~nm}$ and at $652 \mathrm{~nm}$. This is confirmed by the evaluation of the QNAM contrast. In this regard, the QNAM contrast of the $\mathrm{MoS}_{2} \mathrm{ML}$ at $540 \mathrm{~nm}$ is $2.2 \%$, and it decreases drastically to $0.2 \%$ considering the $\mathrm{CL}$ emission at $652 \mathrm{~nm}$. The fainter contrast at the $652 \mathrm{~nm}$ emission in comparison with the $540 \mathrm{~nm}$ is demonstrated by the comparison of the monochromatic QNAM maps reported in Figure 4d,e where the $\mathrm{MoS}_{2} \mathrm{ML}$ contrast is more evident in the $540 \mathrm{~nm}$ QNAM map than in $652 \mathrm{~nm}$. It is worth noting that the bilayer terraces, visible in the panchromatic map, are also made evident in the $540 \mathrm{~nm}$ map, but they do not appear in the $652 \mathrm{~nm}$ map. The difference of the CL spectra is reported in Figure S10, revealing the appearance of the absorption of $\mathrm{MoS}_{2} \mathrm{ML}$ related to the $\mathrm{A}$ and $\mathrm{B}$ excitons.

We demonstrate now how QNAM can also be employed to characterize the optical properties of rotational homostructures. Figure 5 reports the high-resolution analysis carried out 
on $\mathrm{MoS}_{2} 0^{\circ}$ and $60^{\circ}$ rotated bilayers and trilayer terraces. Figure 5a shows the SE image of the analyzed area, in which the presence of $\mathrm{MoS}_{2} \mathrm{ML}$ is clear (red arrow, number II). $0^{\circ}$ and $60^{\circ}$ twisted $\mathrm{MoS}_{2}$ homostructures are indicated by cyan (III) and green (IV) arrows, respectively. The trilayer terraces, twisted by $0^{\circ}$ and $60^{\circ}$, are highlighted by purple (V) and blue (VI) arrows. For trilayer terraces, the twisting angles refer to both the second and third layers. The combined QNAM map of 540 and $650 \mathrm{~nm} \mathrm{CL}$ emissions is reported in Figure 5b. QNAM allows the comparison of the absorption yield of the $0^{\circ}$ and $60^{\circ}$ bilayer and trilayer terraces. As expected and previously reported, ${ }^{34}$ increasing the number of layers results in an enhanced absorption of the two-dimensional semiconducting materials. Interestingly, it is possible to quantify the absorption by means of the QNAM contrast. In the case of the $0^{\circ}$ and $60^{\circ}$ rotated bilayer terrace the QNAM contrast at 540 $\mathrm{nm}$ is $3.2 \%$ and $2.8 \%$, respectively. Meanwhile the QNAM absorption at $652 \mathrm{~nm}$ is exactly $0.5 \%$ for both the bilayer terraces. In the case of the trilayer terraces, the QNAM contrast at $540 \mathrm{~nm}$ is $4 \%$ and $3 \%$ for a rotation angle of $0^{\circ}$ and $60^{\circ}$, respectively. The CL spectra of the different areas indicated by the arrows are reported with the same color code in Figure S11 of the Supporting Information. This analysis demonstrates that $0^{\circ}$ rotated bilayer and trilayer terraces have slightly higher absorption yield in comparison with the $60^{\circ}$ rotated ones; this can be due to the stronger spin-orbit coupling induced band splitting in $0^{\circ}$ twisted structures. ${ }^{57}$ Previous work ${ }^{24}$ has also predicted the modification of the electronic and the optical properties of $\mathrm{MoS}_{2}$ bilayer changing the twisting angle, mainly due to the modification of the interlayer distance of the monolayers, with a maximized effect at $0^{\circ}$ and $60^{\circ}$ twisting angles.

In conclusion, we have developed a novel nondestructive and noncontact technique for mapping the absorption properties of $2 \mathrm{D}$ materials employing the $\mathrm{CL}$ emission of the underlying substrate. One important advantage of this technique is that different substrates have CL emission in different spectral ranges, allowing the quantitative absorption evaluation in such ranges. For instance, sapphire provides information in the deep UV range due to emission of the oxygen vacancy at $323 \mathrm{~nm}(4.3 \mathrm{eV})$; meanwhile, $\mathrm{SiO}_{2} / \mathrm{Si}$ substrate gives information on the whole visible range due to defect-related CL emissions $(1.5-2.7 \mathrm{eV})$. The quantitative nanoscale absorption mapping proves to be versatile in analyzing two different 2-dimensional semiconducting materials, as $\mathrm{MoS}_{2}$ and $\mathrm{MoSe}_{2}$ monolayers on sapphire substrate. Moreover, it has been shown that QNAM can elucidate important novel aspects of $2 \mathrm{D}$ material optical phenomena, as the interlayer interaction in van der Waals heterostructures. First, QNAM demonstrates that the UV absorption is enhanced in $\mathrm{MoSe}_{2} / \mathrm{MoS}_{2} \mathrm{vdW}$ heterostructures. Second, it shows the UV absorption enhancement at tilted grain boundaries with high misorientation angles with respect to mirror twin boundaries. When QNAM is carried out employing $\mathrm{SiO}_{2} / \mathrm{Si}$ as a substrate, it gives insights about the TMD optical properties in the visible range, such as the absorption of $\mathrm{MoS}_{2}$ at different wavelengths. In this case, QNAM allows also the demonstration of the higher absorption yield of the $0^{\circ}$ twisted $\mathrm{MoS}_{2}$ homostructures with respect to the ones with $60^{\circ}$ twisting angle.

Methods. $\mathrm{MoS}_{2}$ and $\mathrm{MoSe}_{2}$ Monolayers on Sapphire Substrates. The monolayer molybdenum disulfide $\left(\mathrm{MoS}_{2}\right)$ and molybdenum diselenide $\left(\mathrm{MoSe}_{2}\right)$ samples have been obtained by the CVD method on highly polished sapphire substrates. ${ }^{43}$ Prior to the growth, the substrates were cleaned by acetone/isopropanol/DI water and further annealed at $1000{ }^{\circ} \mathrm{C}$ in air for $1 \mathrm{~h}$ (DI, deionized). The growth process is based on the gas-phase reaction between $\mathrm{MoO}_{3}(\geq 99.998 \%$ purity, Alfa Aesar) and sulfur/selenium evaporated from solid phase ( $\geq 99.99 \%$ purity, Sigma-Aldrich). A crucible, containing $\sim 5 \mathrm{mg}$ of $\mathrm{MoO}_{3}$ with the sapphire substrates placed facedown above it, was loaded into a $32 \mathrm{~mm}$ outer diameter quartz tube placed in a three-zone furnace. A second crucible located upstream from the growth substrates contained $350 \mathrm{mg}$ of sulfur or $150 \mathrm{mg}$ of selenium. Ultra-high-purity argon (Ar) was used as the carrier gas, and CVD growth was performed at atmospheric pressure. The recipe for the $\mathrm{MoS}_{2}$ growth is as follows: ramp the temperature to $300{ }^{\circ} \mathrm{C}(200 \mathrm{sccm}$ of $\mathrm{Ar}$ flow) and set at $300{ }^{\circ} \mathrm{C}$ for $10 \mathrm{~min}$; ramp to $700{ }^{\circ} \mathrm{C}$ with a 50 ${ }^{\circ} \mathrm{C} / \mathrm{min}$ rate $(10 \mathrm{sccm}$ of $\mathrm{Ar})$ and set at $700{ }^{\circ} \mathrm{C}$ for $10 \mathrm{~min}$, cool down to $570{ }^{\circ} \mathrm{C}$, and open the furnace for rapid cooling (increase the Ar flow to $200 \mathrm{sccm}$ ). For $\mathrm{MoSe}_{2}$, in addition to $10 \mathrm{sccm}$ of $\mathrm{Ar}, 3 \mathrm{sccm}$ of $\mathrm{H}_{2}$ was introduced during $10 \mathrm{~min}$ of growth at $700{ }^{\circ} \mathrm{C}$. More details concerning the growth can be found in the Supporting Information of ref 43.

Transfer Process for vdW Heterostructures. The sample with $\mathrm{MoS}_{2} / \mathrm{MoSe}_{2}$ vdW heterostructures was obtained by two separate transfer steps using a wet transfer $\mathrm{KOH}$ method. First of all, the upper- $\mathrm{MoS}_{2}$ was transferred onto the as-grown $\mathrm{MoSe}_{2}$ monolayer on sapphire. ${ }^{24,34}$ For the transfer, sapphire chips were first spin-coated with PMMA 950 at $1500 \mathrm{rpm}$ for $60 \mathrm{~s}$ and baked at $180{ }^{\circ} \mathrm{C}$ for $5 \mathrm{~min}$. The films were detached in $\mathrm{KOH}(30 \%)$ at moderate temperatures $\left(70{ }^{\circ} \mathrm{C}\right)$, washed three times in deionized water, transferred onto sapphire, and dried at $50{ }^{\circ} \mathrm{C}$ for $30 \mathrm{~min}$. The PMMA was removed by dipping the sample in acetone for $12 \mathrm{~h}$, followed by rinsing with isopropanol and drying in a $\mathrm{N}_{2}$ flow. Such a method provides a polymer clean interface of stacks and minimal damage of material caused by the transfer process.

$\mathrm{MoS}_{2}$ Monolayers on $\mathrm{SiO}_{2} / \mathrm{Si}$ Substrates. Monolayers of $\mathrm{MoS}_{2}$ were grown by CVD on a p-type Si substrate with a 300 $\mathrm{nm}$ thick $\mathrm{SiO}_{2}$ layer at a temperature of $\sim 715^{\circ} \mathrm{C}$. The substrate was placed face-up at the center of a tube furnace on a combustion boat with $10 \mathrm{mg}$ of $\mathrm{MoO}_{3}$ powder spread along the bottom. An identical boat containing $1 \mathrm{~g}$ of sulfur was placed at a position upstream at the opening of the furnace such that the maximum temperature at that position was $250{ }^{\circ} \mathrm{C}$. After the tube was sealed, ultra-high-purity argon gas was allowed to flow at room temperature for $10 \mathrm{~min}$ in order to purge any remaining oxygen. The furnace was then heated to $715^{\circ} \mathrm{C}$ over a period of $25 \mathrm{~min}$ at which point the temperature was held constant for an additional $15 \mathrm{~min}$. The furnace was then allowed to cool back to room temperature naturally over a period of $\sim 90 \mathrm{~min}$. During the entire growth, $\mathrm{Ar}$ was flowed at a rate of $50 \mathrm{sccm}$. For the postgrowth annealing experiments, one sample was subdivided into three, and they were then placed on quartz slides in a tube furnace and annealed at 150,200 , and $250^{\circ} \mathrm{C}$, separately, for $1 \mathrm{~h}$ in $\mathrm{Ar}$ flowing at $50 \mathrm{sccm}$. The samples were cooled naturally to room temperature following the annealing.

Monte Carlo Simulation. The Monte Carlo simulations are carried out with the CASINO software. ${ }^{58}$ The simulated beam consists of $1 \times 10^{4}$ electrons with a spot size of $25 \mathrm{~nm}$ for an accelerating voltage of 2 and $5 \mathrm{kV}$. In order to evaluate the beam interaction, the single layer thickness is set to $0.7 \mathrm{~nm}$. The density of $\mathrm{MoS}_{2}$ and $\mathrm{MoSe}_{2}$ is $\rho=5.06 \mathrm{~g} / \mathrm{cm}^{3}$ and $\rho=6.9$ 
$\mathrm{g} / \mathrm{cm}^{3}$, respectively. The total of the scattering events, employed in the calculation of the percentage of Figure 1b,c, is $1 \times 10^{6}$. In the Monte Carlo simulation the two substrates, employed in this study, i.e., $\mathrm{SiO}_{2} / \mathrm{Si}$ and sapphire, are taken into account. The different density of these materials $\left(\mathrm{SiO}_{2} \rho=\right.$ $2.65 \mathrm{~g} / \mathrm{cm}^{3}$, sapphire $\rho=3.99 \mathrm{~g} / \mathrm{cm}^{3}$ ) does not affect the interaction of the primary beam with the two-dimensional crystal.

Quantitative Absorption Mapping in QNAM. An Attolight Rosa SEM-CL microscope was used for the QNAM experiments. The QNAM experiments are standard CL maps, where the CL signal is employed to evaluate the absorption of the two-dimensional material on top of the light emitting substrate. In the QNAM maps, the CL signal is renormalized in order to maximize the contrast, setting as zero the maximum CL signal coming from the substrate, while the darkest point of the map is set to unity (maximum absorption). ${ }^{59}$ For a quantitative comparison between different areas of the sample, such as between bare and coated substrate regions, the intensity is normalized over the number of pixels that composes each area. All the QNAM maps were obtained at room temperature under UHV conditions. The e-beam current is about $1 \mathrm{nA}$ with an accelerating voltage of 2 or $5 \mathrm{kV}$. The CL signal is sent to a spectrometer with $32 \mathrm{~cm}$ of focal length by means of an objective (numerical aperture, N.A. 0.71) placed in the electron microscope. The system is equipped with a Peltiercooled charge-coupled device (CCD) and $600 \mathrm{l} / \mathrm{mm}$ diffraction grating. The panchromatic QNAM maps are obtained by integrating the CL signal on the whole wavelength range, while the analysis of the absorption at a certain wavelength, namely, monochromatic QNAM maps, was obtained by means of the integrated intensity of each the CL peak evaluated by Gaussian deconvolution. It is worth noting that this difference stands only in the case of multipeak CL emission, as in QNAM analysis of $\mathrm{MoS}_{2}$ on silicon oxides. In fact, in the case of a single peak CL emission, like QNAM analysis on sapphire substrate, panchromatic and monochromatic analyses give exactly the same results.

The quantitative analysis of the flake absorption is carried out by evaluating the contrast between the substrate and the flake. The contrast is calculated by means of formula 1 . The formula is similar to the one employed in the electron-beaminduced current technique for calculating the carrier recombination of extended defects. ${ }^{60}$

$$
C=-\left(\frac{I_{\text {flake }}-I_{\text {substrate }}}{I_{\text {substrate }}}\right) \times 100
$$

In this particular case $I_{\text {substrate }}$ and $I_{\text {flake }}$ are the integrated intensity of the CL signal on the bare substrate and on the flake, respectively. In the case of the analysis on sapphire substrate, the integrated intensity is evaluated by performing the Gaussian deconvolution of each CL emission for both sapphire and $\mathrm{SiO}_{2} / \mathrm{Si}$ analyses. The uncertainty of the QNAM contrast is evaluated to be $0.1 \%$ when it is calculated by means of the integrated intensity of the peak due to the high signal-tonoise ratio of the CCD detector employed in the analysis. All the QNAM contrast values obtained throughout this Letter are summarized in Table S1 of the Supporting Information.

\section{ASSOCIATED CONTENT}

\section{Supporting Information}

The Supporting Information is available free of charge at https://pubs.acs.org/doi/10.1021/acs.nanolett.9b04304.

Details on Monte Carlo simulations, additional QNAM analyses, PL intensity mapping, AFM analysis, and CL spectra (PDF)

Video S1: summary of the physical process of light absorption by the $2 \mathrm{D}$ crystal with nanoscale resolution (AVI)

\section{AUTHOR INFORMATION}

\section{Corresponding Authors}

*E-mail: filippo.fabbri@nano.cnr.it.

*E-mail: anna.fontcuberta-morral@epfl.ch.

ORCID

Andras Kis: 0000-0002-3426-7702

Filippo Fabbri: 0000-0003-1142-0441

Anna Fontcuberta i Morral: 0000-0002-5070-2196

\section{Present Addresses}

${ }^{\nabla}$ D.D.: Department of Quantum Matter Physics, Université de Genève, CH-1211 Geneva, Switzerland

OV.S.: Physics Department, Penn State University, University Park, PA 16802, USA

\section{Author Contributions}

M.N. and L.F. carried the CL experiments and analyzed the QNAM data. D.D. contributed in AFM analysis. D.K., M.B., and V.S. provided the two-dimensional materials on $\mathrm{SiO}_{2} / \mathrm{Si}$ substrates. D.D. and A.K. provided the two-dimensional materials on sapphire substrates. F.F. performed the PL analysis and the Monte Carlo simulations and developed the quantitative analysis. F.F., G.S., and A.F.i.M. provided guidance in all the experiments and manuscript writing. All authors discussed the results and commented on the manuscript.

\section{Notes}

The authors declare no competing financial interest.

\section{ACKNOWLEDGMENTS}

L.F. and A.F.i.M. are thankful for the funding from SNSF via the NCCR QSIT. D.D. and A.K. acknowledge funding from Swiss SNF Sinergia Grant 147607, Marie Curie ITN network "MoWSeS" (grant 317451), and the CCMX Materials Challenge grant "Large Area Growth of 2D Materials for device integration". M.N., M.B., and F.F. want to thank G. Attolini and F. Rossi (IMEM-CNR) for the useful discussions.

\section{REFERENCES}

(1) Lv, R.; Robinson, J. A.; Schaak, R. E.; Sun, D.; Sun, Y.; Mallouk, T. E.; Terrones, M. Transition Metal Dichalcogenides and Beyond: Synthesis, Properties, and Applications of Single- and Few-Layer Nanosheets. Acc. Chem. Res. 2015, 48 (1), 56-64.

(2) Wang, Q. H.; Kalantar-Zadeh, K.; Kis, A.; Coleman, J. N.; Strano, M. S. Electronics and optoelectronics of two-dimensional transition metal dichalcogenides. Nat. Nanotechnol. 2012, 7 (11), 699-712.

(3) Fiori, G.; Bonaccorso, F.; Iannacone, G.; Palacios, T.; Neumaier, D.; Seabaugh, A.; Banerjee, S. K.; Colombo, L. Electronics based on two-dimensional materials. Nat. Nanotechnol. 2014, 9 (10), 768-779.

(4) Xia, F.; Wang, H.; Xiao, D.; Dubey, M.; Ramasubramaniam, A. Two-dimensional material nanophotonics. Nat. Photonics 2014, 8 (12), 899-907. 
(5) Radisavljevic, B.; Radenovic, A.; Brivio, J.; Giacometti, V.; Kis, A. Single-layer $\mathrm{MoS}_{2}$ transistors. Nat. Nanotechnol. 2011, 6 (3), 147150.

(6) Bertolazzi, S.; Krasnozhon, D.; Kis, A. Nonvolatile Memory Cells Based on $\mathrm{MoS}_{2}$ /Graphene Heterostructures. ACS Nano 2013, 7 (4), 3246-3252.

(7) Radisavljevic, B.; Whitwick, M. B.; Kis, A. Integrated Circuits and Logic Operations Based on Single-Layer $\mathrm{MoS}_{2}$. ACS Nano 2011, 5 (12), 9934-9938.

(8) Wang, H.; Yu, L.; Lee, Y. H.; Shi, Y.; Hsu, A.; Chin, M. L.; Li, L. J.; Dubey, M.; Kong, J.; Palacios, T. Integrated Circuits Based on Bilayer $\mathrm{MoS}_{2}$ Transistors. Nano Lett. 2012, 12 (9), 4674-4680.

(9) Mak, K. F.; Lee, C.; Hone, J.; Shan, J.; Heinz, T. F. Atomically Thin $\mathrm{MoS}_{2}$ : A New Direct-Gap Semiconductor. Phys. Rev. Lett. 2010, 105 (13), 136805.

(10) Splendiani, A.; Sun, L.; Zhang, Y.; Li, T.; Kim, J.; Chim, C. Y.; Galli, G.; Wang, F. Emerging photoluminescence in monolayer $\mathrm{MoS}_{2}$. Nano Lett. 2010, 10 (4), 1271-1275.

(11) Buscema, M.; Island, J. O.; Groenendijk, D. J.; Blanter, S. I.; Steele, G. A.; van der Zant, H. S. J.; Castellanos-Gomez, A. Photocurrent generation with two-dimensional van der Waals semiconductors. Chem. Soc. Rev. 2015, 44 (11), 3691-3718.

(12) Geim, A. K.; Grigorieva, I. V. Van der Waals heterostructures. Nature 2013, 499, 419-425.

(13) Courtade, E.; Han, B.; Nakhaie, S.; Robert, C.; Marie, X.; Renucci, P.; Taniguchi, T.; Watanabe, K.; Geelhaar, L.; Lopes, J. M. J.; Urbaszek, B. Spectrally narrow exciton luminescence from monolayer $\mathrm{MoS}_{2}$ and $\mathrm{MoSe}_{2}$ exfoliated onto epitaxially grown hexagonal BN. Appl. Phys. Lett. 2018, 113, 032106.

(14) Cadiz, F.; Courtade, E.; Robert, C.; Wang, G.; Shen, Y.; Cai, H.; Taniguchi, T.; Watanabe, K.; Carrere, H.; Lagarde, D.; Manca, M.; Amand, T.; Renucci, P.; Tongay, S.; Marie, X.; Urbaszek, B. Excitonic linewidth approaching the homogeneous limit in $\mathrm{MoS}_{2^{-}}$ based van der Waals heterostructures. Phys. Rev. X 2017, 7, 021026.

(15) Lee, C.-H.; Lee, G.-H.; van der Zande, A. M.; Chen, W.; Li, Y.; Han, M.; Cui, X.; Arefe, G.; Nuckolls, C.; Heinz, T. F.; Guo, J.; Hone, J.; Kim, P. Atomically thin $\mathrm{p}-\mathrm{n}$ junctions with van der Waals heterointerfaces. Nat. Nanotechnol. 2014, 9, 676-681.

(16) Ceballos, F.; Bellus, M. Z.; Chiu, H.-Y.; Zhao, H. Ultrafast charge separation and indirect exciton formation in a $\mathrm{MoS}_{2}-\mathrm{MoSe}_{2}$ van der Waals heterostructure. ACS Nano 2014, 8, 12717-12724.

(17) Ponomarev, E.; Ubrig, N.; Gutiérrez-Lezama, I.; Berger, H.; Morpurgo, A. F. Semiconducting van der Waals interfaces as artificial semiconductors. Nano Lett. 2018, 18, 5146-5152.

(18) Rivera, P.; Yu, H.; Seyler, K. L.; Wilson, N. P.; Yao, W.; Xu, X. Interlayer valley excitons in heterobilayers of transition metal dichalcogenides. Nat. Nanotechnol. 2018, 13, 1004-1015.

(19) Jin, C.; Ma, E. Y.; Karni, O.; Regan, E. C.; Wang, F.; Heinz, T. F. Ultrafast dynamics in van der Waals heterostructures. Nat. Nanotechnol. 2018, 13, 994-1003.

(20) Ovesen, S.; Brem, S.; Linderälv, C.; Kuisma, M.; Korn, T.; Erhart, P.; Selig, M.; Malic, E. Interlayer exciton dynamics in van der Waals heterostructures. Commun. Phys. 2019, 2, 23.

(21) Gong, C.; Zhang, H.; Wang, W.; Colombo, L.; Wallace, R. M.; Cho, K. Band alignment of two-dimensional transition metal dichalcogenides: Application in tunnel field effect transistors. Appl. Phys. Lett. 2013, 103 (5), 053513.

(22) Ross, J. S.; Rivera, P.; Schaibley, J.; Lee-Wong, E.; Yu, H.; Taniguchi, T.; Watanabe, K.; Yan, J.; Mandrus, D.; Cobden, D.; Yao, $\mathrm{W}$.; Xu, X. Interlayer exciton optoelectronics in a $2 \mathrm{D}$ heterostructure $\mathrm{p}-\mathrm{n}$ junction. Nano Lett. 2017, 17, 638-643.

(23) Nayak, P. K.; Horbatenko, Y.; Ahn, S.; Kim, G.; Lee, J.U.; Yeol Ma, K.; Jang, A.-R.; Lim, H.; Kim, D.; Ryu, S.; Cheong, H.; Park, N.; Shin, H. S. Probing evolution of twist-angle-dependent interlayer excitons in $\mathrm{MoSe}_{2} / \mathrm{WSe}_{2}$ van der Waals heterostructures. ACS Nano 2017, 11 (4), 4041-4050.

(24) Liu, K.; Zhang, L.; Cao, T.; Jin, C.; Qiu, D.; Zhou, Q.; Zettl, A.; Yang, P.; Louie, S. G.; Wang, F. Evolution of interlayer coupling in twisted molybdenum disulfide bilayers. Nat. Commun. 2014, 5, 4966.
(25) Zhang, N.; Surrente, A.; Baranowski, M.; Maude, D. K.; Gant, P.; Castellanos-Gomez, A.; Plochocka, P. Moiré intralayer excitons in a $\mathrm{MoSe}_{2} / \mathrm{MoS}_{2}$ heterostructure. Nano Lett. 2018, 18, 7651-7657.

(26) Tran, K.; Moody, G.; Wu, F.; Lu, X.; Choi, J.; Kim, K.; Rai, A.; Sanchez, D. A.; Quan, J.; Singh, A.; et al. Evidence for moiré excitons in van der Waals heterostructures. Nature 2019, 567, 71-75.

(27) Mak, K. F.; He, K.; Lee, C.; Hyoung Lee, G.; Hone, J.; Heinz, T. F.; Shan, J. Tightly bound trions in monolayer $\mathrm{MoS}_{2}$. Nat. Mater. 2013, 12, 207-211.

(28) Ross, J. S.; Wu, S.; Yu, H.; Ghimire, N. J.; Jones, A. M.; Aivazian, G.; Yan, J.; Mandrus, D. G.; Xiao, D.; Yao, W.; Xu, X. Electrical control of neutral and charged excitons in a monolayer semiconductor. Nat. Commun. 2013, 4, 1474.

(29) Jones, A. M.; Yu, H.; Ghimire, N. J.; Wu, S.; Aivazian, G.; Ross, J. S.; Zhao, B.; Yan, J.; Mandrus, D. G.; Xiao, D.; Yao, W.; Xu, X. Optical generation of excitonic valley coherence in monolayer $\mathrm{WSe}_{2}$. Nat. Nanotechnol. 2013, 8, 634-638.

(30) Mak, K. F.; He, K.; Shan, J.; Heinz, T. F. Control of valley polarization in monolayer $\mathrm{MoS}_{2}$ by optical helicity. Nat. Nanotechnol. 2012, 7, 494-498.

(31) Zeng, H.; Dai, J.; Yao, W.; Xiao, D.; Cui, X. Valley polarization in $\mathrm{MoS}_{2}$ monolayers by optical pumping. Nat. Nanotechnol. 2012, 7, 490-493.

(32) van der Zande, A. M.; Huang, P. Y.; Chenet, D. A.; Berkelbach, T. C.; You, Y. M.; Lee, G.-H.; Heinz, T. F.; Reichman, D. R.; Muller, D. A.; Hone, J. C. Grains and grain boundaries in highly crystalline monolayer molybdenum disulphide. Nat. Mater. 2013, 12, 554-561.

(33) Dhakal, K. P.; Duong, D. L.; Lee, J.; Nam, H.; Kim, M.; Kan, M.; Lee, Y. H.; Kim, J. Confocal absorption spectral imaging of MoS 2 : optical transitions depending on the atomic thickness of intrinsic and chemically doped $\mathrm{MoS}_{2}$. Nanoscale 2014, 6, 13028-13035.

(34) Castellanos-Gomez, A.; Quereda, J.; Van der Meulen, H. P.; Agraï, N.; Rubio-Bollinger, G. Spatially resolved optical absorption spectroscopy of single- and few-layer $\mathrm{MoS}_{2}$ by hyperspectral imaging. Nanotechnology 2016, 27, 115705.

(35) Bao, W.; Borys, N. J.; Ko, C.; Suh, J.; Fan, W.; Thron, A.; Zhang, Y.; Buyanin, A.; Zhang, J.; Cabrini, S.; et al. Visualizing nanoscale excitonic relaxation properties of disordered edges and grain boundaries in monolayer molybdenum disulfide. Nat. Commun. 2015, 6, 7993.

(36) Lee, Y.; Park, S.; Kim, H.; Han, G. H.; Lee, Y. H.; Kim, J. Characterization of the structural defects in CVD-grown monolayered $\mathrm{MoS}_{2}$ using near-field photoluminescence imaging. Nanoscale 2015, 7, 11909-11914.

(37) Su, W.; Kumar, N.; Mignuzzi, S.; Crain, J.; Roy, D. Nanoscale mapping of excitonic processes in single-layer $\mathrm{MoS}_{2}$ using tipenhanced photoluminescence microscopy. Nanoscale 2016, 8, 1056410569.

(38) Okuno, Y.; Lancry, O.; Tempez, A.; Cairone, C.; Bosi, M.; Fabbri, F.; Chaigneau, M. Probing the nanoscale light emission properties of a CVD-grown $\mathrm{MoS}_{2}$ monolayer by tip-enhanced photoluminescence. Nanoscale 2018, 10, 14055-14059.

(39) Park, K.-D.; Khatib, O.; Kravtsov, V.; Clark, G.; Xu, X.; Raschke, M. B. Hybrid Tip-Enhanced Nanospectroscopy and Nanoimaging of Monolayer WSe ${ }_{2}$ with Local Strain Control. Nano Lett. 2016, 16, 2621-2627.

(40) Fabbri, F.; Rotunno, E.; Cinquanta, E.; Campi, D.; Bonnini, E.; Kaplan, D.; Lazzarini, L.; Bernasconi, M.; Ferrari, C.; Longo, M.; et al. Novel near-infrared emission from crystal defects in $\mathrm{MoS}_{2}$ multilayer flakes. Nat. Commun. 2016, 7, 13044.

(41) Zheng, S.; So, J.-K.; Liu, F.; Liu, Z.; Zheludev, N.; Fan, H. J. Giant Enhancement of Cathodoluminescence of Monolayer Transitional Metal Dichalcogenides Semiconductors. Nano Lett. 2017, 17, 6475-6480.

(42) Raja, A.; Chaves, A.; Yu, J.; Arefe, G.; Hill, H. M.; Rigosi, A. F.; Berkelbach, T. C.; Nagler, P.; Schüller, C.; Korn, T.; et al. Coulomb engineering of the bandgap and excitons in two-dimensional materials. Nat. Commun. 2017, 8, 15251. 
(43) Dumcenco, D.; Ovchinnikov, D.; Marinov, K.; Lazić, P.; Gibertini, M.; Marzari, N.; Lopez Sanchez, O.; Kung, Y.-C.; Krasnozhon, D.; Chen, M.-W.; et al. Large-Area Epitaxial Monolayer $\mathrm{MoS}_{2}$. ACS Nano 2015, 9 (4), 4611-4620.

(44) Pezzotti, G.; Wan, K.; Munisso, M. C.; Zhu, W. Stress dependence of $\mathrm{F}+$ center cathodoluminescence of sapphire. Appl. Phys. Lett. 2006, 89 (4), 041908.

(45) Ghamnia, M.; Jardin, C.; Bouslama, M. Luminescent centres F and $\mathrm{F}+$ in $\alpha$-alumina detected by cathodoluminescence technique. J. Electron Spectrosc. Relat. Phenom. 2003, 133, 55-63.

(46) Dong, N.; Li, Y.; Feng, Y.; Zhang, S.; Zhang, X.; Chang, C.; Fan, J.; Zhang, L.; Wang, J. Optical Limiting and Theoretical Modelling of Layered Transition Metal Dichalcogenide Nanosheets. Sci. Rep. 2015, 5, 14646.

(47) Li, W.; Birdwell, A. G.; Amani, M.; Burke, R. A.; Ling, X.; Lee, Y.-H.; Liang, X.; Peng, L.; Richter, C. A.; Kong, J.; et al. Broadband optical properties of large-area monolayer CVD molybdenum disulfide. Phys. Rev. B: Condens. Matter Mater. Phys. 2014, 90, 195434. (48) Zhang, Y.; Chang, T.-R.; Zhou, B.; Cui, Y.-T.; Yan, H.; Liu, Z.; Schmitt, F.; Lee, J.; Moore, R.; Chen, Y.; et al. Direct observation of the transition from indirect to direct bandgap in atomically thin epitaxial $\mathrm{MoSe}_{2}$. Nat. Nanotechnol. 2014, 9, 111-115.

(49) Park, H. G.; Kim, T. J.; Ullah, F.; Le, V. L.; Nguyen, H. T.; Kim, Y. S.; Kim, Y. D. Temperature Dependence of the Dielectric Function of Monolayer $\mathrm{MoSe}_{2}$. Sci. Rep. 2018, 8, 3173.

(50) Rigosi, A. F.; Hill, H. M.; Li, Y.; Chernikov, A.; Heinz, T. F. Probing Interlayer Interactions in Transition Metal Dichalcogenide Heterostructures by Optical Spectroscopy: $\mathrm{MoS}_{2} / \mathrm{WS}_{2}$ and $\mathrm{MoSe}_{2} /$ $\mathrm{WSe}_{2}$. Nano Lett. 2015, 15, 5033-5038.

(51) Wurstbauer, U.; Miller, B.; Parzinger, E.; Holleitner, A. W. Light matter interaction in transition metal dichalcogenides and their heterostructures. J. Phys. D: Appl. Phys. 2017, 50, 173001.

(52) Najmaei, S.; Liu, Z.; Zhou, W.; Zou, X.; Shi, G.; Lei, S.; Yakobson, B. I.; Idrobo, J.-C.; Ajayan, P. M.; Lou, J. Vapour phase growth and grain boundary structure of molybdenum disulphide atomic layers. Nat. Mater. 2013, 12, 754-759.

(53) Ly, T. H.; Perello, D. J.; Zhao, J.; Deng, Q.; Kim, H.; Han, G. H.; Chae, S. H.; Jeong, H. Y.; Lee, Y. H. Misorientation-angledependent electrical transport across molybdenum disulfide grain boundaries. Nat. Commun. 2016, 7, 10426.

(54) Zou, X.; Liu, Y.; Yakobson, B. I. Predicting Dislocations and Grain Boundaries in Two-Dimensional Metal-Disulfides from the First Principles. Nano Lett. 2013, 13, 253-258.

(55) Manzeli, S.; Ovchinnikov, D.; Pasquier, D.; Yazyev, O. V.; Kis, A. 2D transition metal dichalcogenides. Nat. Rev. Mater. 2017, 2, 17033.

(56) Skuja, L. Optically active oxygen-deficiency-related centers in amorphous silicon dioxide. J. Non-Cryst. Solids 1998, 239, 16-48.

(57) Shinde, S. M.; Dhakal, K. P.; Chen, X.; Yun, W. S.; Lee, J. D.; Kim, H.; Ahn, J.-H. Stacking-controllable interlayer coupling and symmetric configuration of multilayered $\mathrm{MoS}_{2}$. NPG Asia Mater. 2018, 10, e468-e468.

(58) Drouin, D.; Couture, A. R.; Joly, D.; Tastet, X.; Aimez, V.; Gauvin, R. CASINO V2.42-A Fast and Easy-to-use Modeling Tool for Scanning Electron Microscopy and Microanalysis Users. Scanning 2007, 29, 92-101.

(59) Hunter, J. D. Matplotlib: A 2D Graphics Environment. Comput. Sci. Eng. 2007, 9, 90-95.

(60) Reimer, L. Scanning Electron Microscopy: Physics of Image Formation and Microanalysis; Springer: Berlin Heidelberg, 1998. 